\title{
From Hubbard bands to spin-polaron excitations in the doped Mott material $\mathrm{Na}_{x} \mathrm{CoO}_{2}$
}

\author{
Aljoscha Wilhelm, ${ }^{1}$ Frank Lechermann, ${ }^{1}$ Hartmut Hafermann, ${ }^{2}$ Mikhail I. Katsnelson, ${ }^{3}$ and Alexander I. Lichtenstein ${ }^{1}$ \\ ${ }^{1}$ I. Institut für Theoretische Physik, Universität Hamburg, D-20355 Hamburg, Germany \\ ${ }^{2}$ Institut de Physique Théorique (IPhT), CEA, CNRS, 91191 Gif-sur-Yvette, France \\ ${ }^{3}$ Radboud University Nijmegen, Institute for Molecules and Materials, NL-6525 AJ Nijmegen, The Netherlands
}

(Received 19 August 2014; revised manuscript received 22 March 2015; published 10 April 2015)

\begin{abstract}
We investigate the excitation spectrum of strongly correlated sodium cobaltate within a realistic many-body description beyond dynamical mean-field theory (DMFT). At lower doping around $x=0.3$, rather close to Mott-critical half-filling, the single-particle spectral function of $\mathrm{Na}_{x} \mathrm{CoO}_{2}$ displays an upper Hubbard band which is captured within DMFT. Momentum-dependent self-energy effects beyond DMFT become dominant at higher doping. Around a doping level of $x \sim 0.67$, the incoherent excitations give way to finite-energy spin-polaron excitations in close agreement with optics experiments. These excitations are a direct consequence of the formation of bound states between quasiparticles and paramagnons in the proximity of in-plane ferromagnetic ordering.
\end{abstract}

DOI: 10.1103/PhysRevB.91.155114

PACS number(s): 71.27.+a, 71.45.-d, 72.10.Di

\section{INTRODUCTION}

The relevance of electronic correlation effects beyond the cuprate paradigm has become obvious in recent years due to issues raised by materials such as cobaltates, iron pnictides/chalcogenides, oxide heterostructures, or iridates. The experimental phase diagram of the quasi-two-dimensional sodium cobaltate system $\mathrm{Na}_{x} \mathrm{CoO}_{2}$ exhibits nearly all hallmarks of strongly correlated physics [see Fig. 1(a)]. Electron doping of stacked triangular $\mathrm{CoO}_{2}$ layers via $x \mathrm{Na}^{+}$ions results in superconductivity upon intercalation with water [1], charge disproportionation [2,3], Curie-Weiss behavior [4], in-plane ferromagnetic (FM) order [5-7], and a regime of large thermopower [8]. The nominal low-spin oxidation state of cobalt in this compound amounts to $\mathrm{Co}^{(4-x)+}$ with occupation $3 d^{5+x}$. At low energy, the electronic states are mainly governed by the $t_{2 g}$ manifold of the $\operatorname{Co}(3 d)$ shell, which becomes completely filled in the band-insulating $x=1$ limit. With a bandwidth $W \sim 1.6 \mathrm{eV}$ [9] and an estimated on-site Hubbard interaction $U$ of $\sim 3-5 \mathrm{eV}[10,11]$, the material is by all means located in the strongly correlated large $U / W$ regime.

The appropriate theoretical tool to deal with strong correlation physics is the dynamical mean-field theory (DMFT). The formation of renormalized quasiparticles, spectral-weight transfer to Hubbard excitations, local-moment behavior, and the Mott transition are well described by means of a local but frequency-dependent electronic self-energy $\Sigma(\omega)$ [12]. In sodium cobaltate, most of the puzzling correlation physics, however, appears in the strongly doped regime for $x \gtrsim 0.6$ [13]. Some experiments suggest that even the superconducting dome occurs in the very same part of the phase diagram because of Co-charging effects via $\mathrm{H}_{2} \mathrm{O}$ [14]. Here the inplane magnetic characteristics change from antiferromagnetic (AFM) tendencies towards FM [3,15]. We will see that, despite the high doping level, nonlocal corrections to DMFT become important. Incorporating the impact of the long-range FM fluctuations around the $\Gamma$ point is naturally hard to achieve in cluster extensions to DMFT [16]. Not so for novel diagrammatic extensions thereof, such as the dual(fermion/boson) [17,18] approaches or DГ A [19]. In this work, we promote these techniques to a new level by combining the dual-fermion method with an ab initio approach. This way, we are able to show that the interaction of quasiparticles with collective magnetic excitations can lead to intriguing many-particle excitations in real materials prone to magnetic order. As our main result, we identify previously observed excitations in correlated $\mathrm{Na}_{x} \mathrm{CoO}_{2}$ close to in-plane FM order as intricate spin-polaron excitations.

\section{REALISTIC LOW-ENERGY MODEL}

Density functional theory in the local density approximation (LDA) is employed for the material-specific part. Several works have shown $[15,20-23]$ that, due to the $t_{2 g}$ filling and trigonal crystal-field effects, the bulk of the essential cobaltate physics may be described already within an interacting effective one-band model of dominant $a_{1 g}$ character. Charge self-consistent combined density functional theory and DMFT calculations within a three-band model confirm the validity of the single-band treatment: At $x=0.3$, the $e_{g}^{\prime}$-like hole pockets are suppressed. Correlations repel the filled bands farther from the Fermi level at high doping $x=0.7$ [24]. To achieve finite doping in the pseudopotential approach, an effective $\mathrm{Na}$ ion with nuclear charge $Z_{\mathrm{Na}}+x$ is used in virtual-crystal LDA calculations for a single-formula-unit cell. Interlayer bonding-antibonding splittings are thereby neglected. For the lattice parameters at $x=0.67$ the values $a=2.83 \AA$ and $c=10.90 \AA$ (given in double-layer counting) are used. We utilize the relaxed LDA value $z_{0}=0.084$ for the $\mathrm{O}$ height as in the work by Singh [9]. Figure 1(b) displays the band structure in the LDA to density functional theory for sodium cobaltate at $x=0.67$. Besides the isolated $t_{2 g}$-like manifold at low energy we readily see the Fermi-surface-forming maximally localized Wannier-like band with dominant $a_{1 g}$-like character. Note that the $e_{g}^{\prime}$-like hole pockets are filled at this doping. To good approximation, the corresponding bands may be ignored for the specific low-energy physics, leading to an effective oneband tight-binding description. For the hopping amplitudes $\left(t, t^{\prime}, t^{\prime \prime}\right)$ up to third-nearest-neighbor $(\mathrm{NN})$ distances we obtain values of $(-178,41,27) \mathrm{meV}$ at $x=0.67$. The real-space Wannier orbital with local $a_{1 g}$ resemblance in Fig. 1(c) has not only significant weight on the nearby oxygen ions. It also displays a mixed $e_{g} / e_{g}^{\prime}$ structure on NN Co ions, rendering the entangled multiorbital contributions obvious. Including 


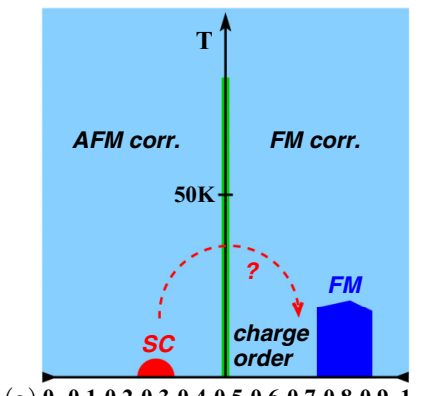

(a) 00.10 .20 .30 .40 .50 .60 .70 .80 .91

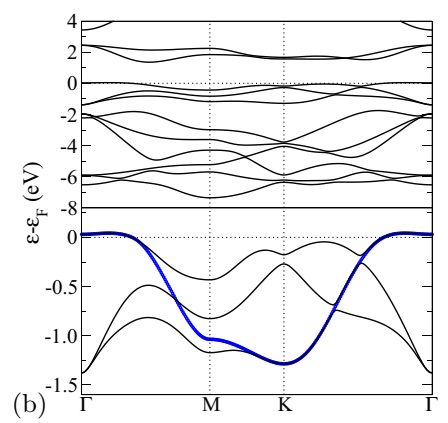

(c)

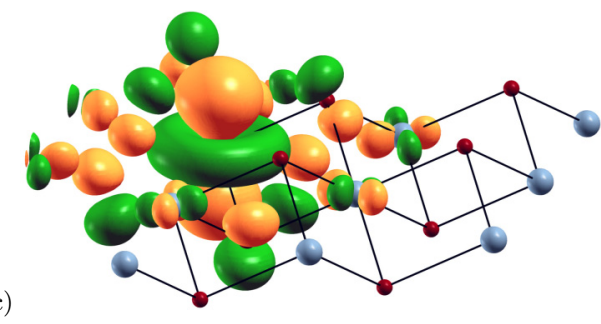

FIG. 1. (Color online) (a) Schematic $T-x$ phase diagram for the $\mathrm{CoO}_{2}$ planes in sodium cobaltate. The green bar at $x=0.5$ marks the insulating charge-ordered state and the dashed arrow indicates the possibility for superconductivity close to $x=0.67$ [14]. (b) LDA band structure for $x=0.67$. The lower panel shows a magnification of the region around $\varepsilon_{\mathrm{F}}$ with the $a_{1 g}$-like Wannier band (blue). (c) Corresponding real-space Wannier orbital in the $\mathrm{CoO}_{2}$ plane (Co, light blue; $\mathrm{O}$, red).

electron hopping up to at least the third-NNs in subsequent many-body treatments of $\mathrm{Na}_{x} \mathrm{CoO}_{2}$ is crucial. The long-range hopping is essential to describe the band flattening close to the $\Gamma$ point, resulting in an extended Van Hove singularity at the upper band edge for the cobaltate $(t<0)$ case. Since we do not expect any qualitative changes, the full Wannier Hamiltonian for $x=0.67$ is used for the kinetic part in the many-body calculations at all investigated doping levels.

With the derived Wannier dispersion $\varepsilon(\mathbf{k})$, the complete interacting problem is cast into an effective one-band Hubbard model on the triangular $\mathrm{CoO}_{2}$ lattice described by the grandcanonical Hamiltonian

$$
H=\sum_{\mathbf{k} \sigma} \varepsilon(\mathbf{k}) c_{\mathbf{k} \sigma}^{\dagger} c_{\mathbf{k} \sigma}+\sum_{i} U n_{i \uparrow} n_{i \downarrow}-\mu \sum_{i} n_{i} .
$$

Here $\mathbf{k}$ denotes quasimomenta and the index $i$ labels the lattice sites. The operators $c_{\sigma}^{(\dagger)}$ denote annihilators (creators) for the Wannier electrons with spin projection $\sigma=\uparrow, \downarrow$ and we write $n_{\sigma}=c_{\sigma}^{\dagger} c_{\sigma}, n=n_{\uparrow}+n_{\downarrow}$. We choose an on-site Coulomb interaction of $U=5 \mathrm{eV}$ [25]. The data discussed in the following are obtained at temperature $T=387 \mathrm{~K}$. At various doping levels $x$, we apply the dual-fermion (DF) approach [17] using ladder summation [26] to solve this realistic many-body problem tailored to the key $\mathrm{Na}_{x} \mathrm{CoO}_{2}$ physics.

\section{DUAL-FERMION METHOD}

We address the many-body problem (1) by means of the DF approach [17]. Using a diagrammatic extension of DMFT makes it possible to describe the nonlocal correlation physics that we expect. The collective two-particle excitations in this approach are described by the Bethe-Salpeter equation (BSE) for the lattice vertex function $\Gamma$, which reads

$$
\left[\tilde{\Gamma}_{v}^{\mathrm{s} / \mathrm{c}}\right]_{\omega \omega^{\prime}}^{-1}(\mathbf{q})=\left[\gamma_{v}^{\mathrm{s} / \mathrm{c}}\right]_{\omega \omega^{\prime}}^{-1}-\tilde{\chi}_{\nu}^{\omega}(\mathbf{q}) \delta_{\omega \omega^{\prime}}
$$

Here $\omega_{n}=(2 n+1) \pi / \beta$ and $v_{m}=2 m \pi / \beta$ denote the fermionic and bosonic Matsubara frequencies and the equation is valid for the spin (s) and charge (c) channels. We use the tilde to indicate that the quantities are defined in terms of DFs. Here $\gamma$ plays the role of the irreducible vertex and is given by the fully connected vertex function of the DMFT impurity model. It is local, but frequency dependent. The frequency dependence is important for the description of the collective excitations when correlations are strong. The BSE generates the sum of ladder diagrams at all orders and describes repeated particle-hole scattering which gives rise to long-wavelength two-particle collective excitations [27].

In DF, we can describe the scattering of single particles with collective excitations through a momentum-dependent (dual) electronic self-energy $\tilde{\Sigma}_{\omega}(\mathbf{k})$,

$$
\tilde{\Sigma}_{\omega}(\mathbf{k})=\sum_{\alpha \nu \omega^{\prime} \mathbf{q}} a_{\alpha} \gamma_{\nu \omega \omega^{\prime}}^{\alpha} \tilde{G}_{\omega+\nu}(\mathbf{k}+\mathbf{q}) \tilde{\chi}_{\nu}^{\omega^{\prime}}(\mathbf{q}) \hat{\Gamma}_{\nu \omega^{\prime} \omega}^{\alpha}(\mathbf{q}) \text {. }
$$

Here we have introduced the shorthand notation $\hat{\Gamma}_{\nu \omega \omega^{\prime}}^{\mathrm{s} / \mathrm{c}}(\mathbf{q})=$ $\tilde{\Gamma}_{\nu \omega \omega^{\prime}}^{\mathrm{s} / \mathrm{c}}(\mathbf{q})-\frac{1}{2} \gamma_{\nu \omega \omega^{\prime}}^{\mathrm{s} / \mathrm{c}}$, where subtracting the local contribution avoids double counting of the second-order contribution. The sum over $\alpha$ runs over the charge and spin channels with $a_{\mathrm{c}}=1 / 2, a_{\mathrm{s}}=3 / 2$. The frequency (momentum) sums are understood to be normalized by the inverse temperature $\beta=1 / T$ and number of $k$ points, respectively. The lattice Green's function, which can be related to observables, is expressed in the following form [18]:

$$
G_{\omega}(\mathbf{k})=\left\{g_{\omega}^{-1}\left[1+g_{\omega} \tilde{\Sigma}_{\omega}(\mathbf{k})\right]^{-1}+\Delta_{\omega}-\varepsilon(\mathbf{k})\right\}^{-1} .
$$

In the above equation, $\tilde{\chi}_{v}^{\omega}(\mathbf{q})=-\sum_{\mathbf{k}} \tilde{G}_{\omega}(\mathbf{k}) \tilde{G}_{\omega+v}(\mathbf{k}+\mathbf{q})$ denotes the dual particle-hole bubble. The bare dual Green's function is given by $\tilde{G}_{\omega}^{0}(\mathbf{k})=G_{\omega}^{\mathrm{DMFT}}(\mathbf{k})-g_{\omega}$. Subtracting the local self-consistent impurity Green's function $g_{\omega}$ from the DMFT lattice Green's function $G_{\omega}^{\mathrm{DMFT}}(\mathbf{k})=\left[g_{\omega}^{-1}+\Delta_{\omega}-\right.$ $\left.\varepsilon_{\mathbf{k}}\right]^{-1}$ efficiently avoids double counting of local contributions.

In our calculations, we start from a converged DMFT solution at given parameters corresponding to the selfconsistency condition $\sum_{\mathbf{k}} \tilde{G}_{\omega}^{0}(\mathbf{k})=0$ [i.e., the diagrammatic correction (3) is not taken into account]. We then compute the local two-particle vertex function $\gamma$, which allows us to evaluate the BSE and dual self-energy correction. The dual Green's functions are self-consistently renormalized. We finally obtain spectral functions by analytical continuation of (4). The impurity self-energy and the local one- and twoparticle Green's functions are computed using an optimized [28] continuous-time hybridization expansion quantum Monte Carlo solver [29] with improved estimators [30] for the self-energy and vertex function. For the Monte Carlo as well as for the DF part for retrieving the interacting Green's functions, we employ a fully parallelized implementation. We exploit the lattice symmetries and compute the diagrams on a $128 \times 128 \times 128$ frequency-lattice grid using the fast-Fouriertransform algorithm. For further details on the DF formalism and dual perturbation theory, see Ref. [31]. 
It is known that charge-ordering effects may influence the triangular hopping paths [32]. However, nonlocal charge correlations are included in the DF approach and two-particle spin and charge susceptibilities are indeed very similar to recent DMFT + vertex results [22]. The principal physics discussed here is not altered by the presence of charge ordering.

\section{RESULTS}

Figure 2 shows the DF and DMFT spectral functions $A_{\omega}(\mathbf{k})=-(1 / \pi) \operatorname{Im} G_{\omega+i 0^{+}}(\mathbf{k})$ in the unoccupied part of the
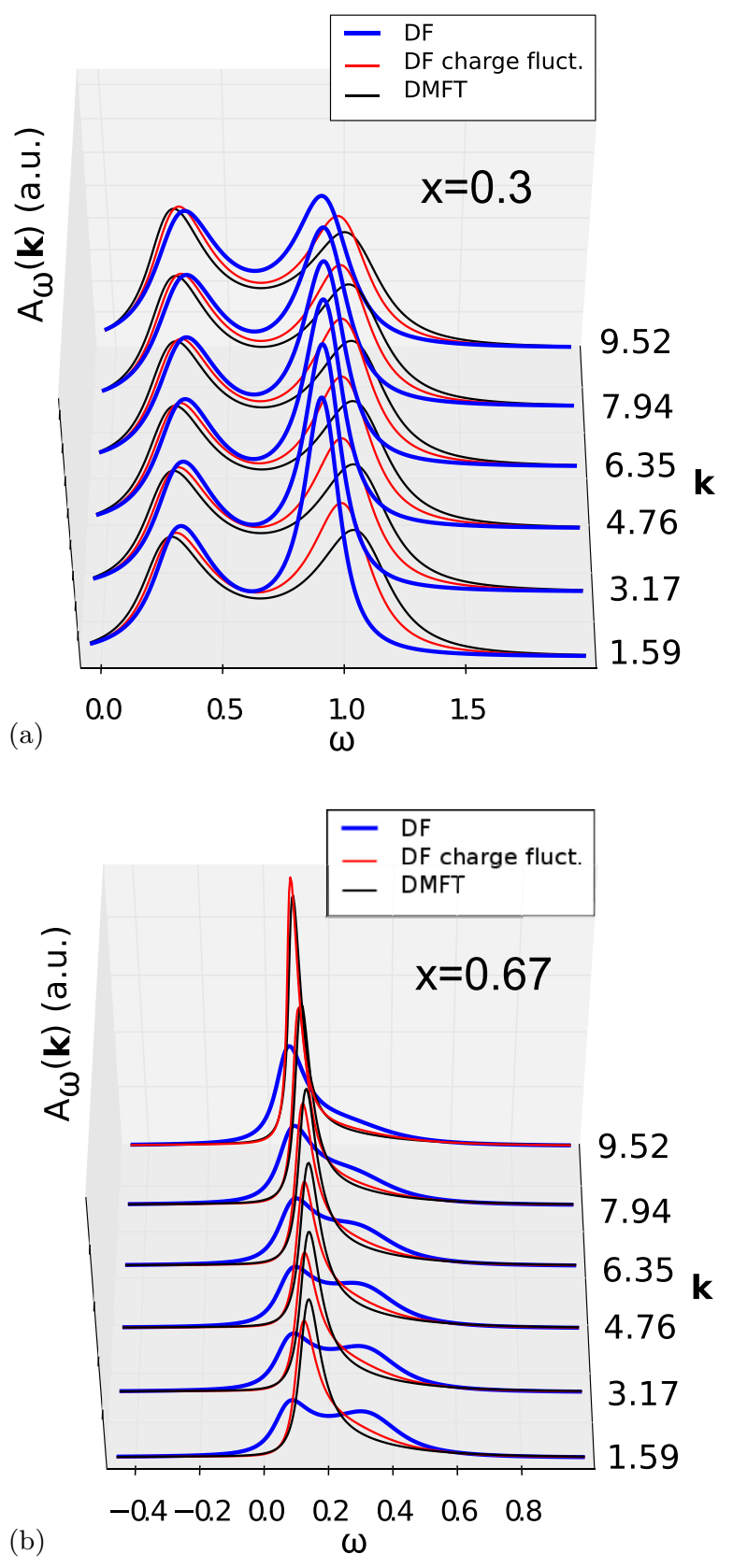

FIG. 2. (Color online) Interacting and momentum-resolved $\mathrm{Na}_{x} \mathrm{CoO}_{2}$ spectral functions close to the $\Gamma$ point in the direction $\Gamma \rightarrow \mathrm{M}$ and in units of $10^{-2}(2 \pi / a)$. The results are for doping levels $x=0.3$ (a) and $x=0.67$ (b). Results for DMFT, DF, and DF restricted to charge fluctuations are shown. The Fermi level is located at $\omega=0$. spectrum $\omega>0$ in reciprocal space and close to the $\Gamma$ point. At low doping, $x=0.3$, relatively close to half filling, both DMFT and DF agree qualitatively rather well, displaying a broadened quasiparticle (QP) peak and an upper Hubbard band located at an energy of $\omega \sim 1 \mathrm{eV}$. Within DF, the upper Hubbard band is shifted to slightly lower energies and considerably narrowed. Because of the diagrammatic construction of the the dual self-energy (3), we can readily separate contributions from collective spin and charge excitations. Restricting the DF calculation to charge fluctuations results in a spectrum that is much closer to DMFT. At this doping, the magnetic susceptibility is peaked at the $K$ point of the Brillouin zone [22], which we confirm in our data. There is hence a tendency to AFM ordering, but the system does not order because of frustration. Instead, the presence of dynamical AFM correlations decreases the fluctuations and leads to an interplay of Slater and Mott physics that increases the coherence of the single-particle excitations. A similar situation occurs in the two-dimensional square lattice [31].

At $x=0.67$, the difference between DMFT and DF is more significant, giving rise to a qualitatively different excitation spectrum above $\varepsilon_{\mathrm{F}}$. In DMFT, the QP peak is considerably larger and the $\mathrm{QP}$ weight $Z$ is significantly enhanced compared to the case of low doping. This is expected, since $Z \approx 1$ should hold far away from half filling and towards the opposite (band-insulating) end point $x=1$. We further see that the upper Hubbard band has completely dissolved in the DMFT perspective. In DF, on the contrary, the QP peak close to $\Gamma$ is strongly renormalized. The spectral function additionally exhibits a broad sideband excitation at $\omega \sim 0.3-0.4 \mathrm{eV}$. By restricting the DF calculation to the charge channel only, this sideband excitation disappears. This is a strong indication that this excitation is of magnetic origin.

A weak absorption feature already present at room temperature has previously been reported from optics experiments of nearly ferromagnetic $\mathrm{Na}_{0.7} \mathrm{CoO}_{2}$ for $x=0.7$ by Wan et al. [33]. The broad feature was observed in the mid-infrared at an energy of $\omega \sim 0.4 \mathrm{eV}$, which is in remarkably good agreement with our result. The authors of that work speculated about spin polarons as one of the possible mechanisms. This interpretation is not unlikely due to an enhanced ferromagnetic susceptibility and the presence of strong FM fluctuations at this doping. We find that the leading eigenvalue of the magnetic channel of the BSEs is largest for $\mathbf{q}=0$, i.e., for ferromagnetic alignment of the spins. This is in line with the paramagnons found in the vicinity of the $\Gamma$ point in a previous study [22].

In elementary theory for the spin-polaron, one considers an electron in a ferromagnetic background and a single magnon in the $s-d$ [34-36] or $t-J$ models [37]. In the latter, the presence of bound states depends crucially on the lattice dimensionality and anomalies in the electronic spectrum. In three dimensions (3D) and small FM $J>0$, there are no spin-polaron bound states in an almost filled band in presence of Nagaoka ferromagnetism [38]. This is similar to the classical Slater-Koster impurity problem, where a weak potential in a 3D crystal does not lead to the formation of a bound state. In 1D, however, a bound state forms at an arbitrarily small ratio of $J / t$ [37]. For the quasi-2D system the situation is 


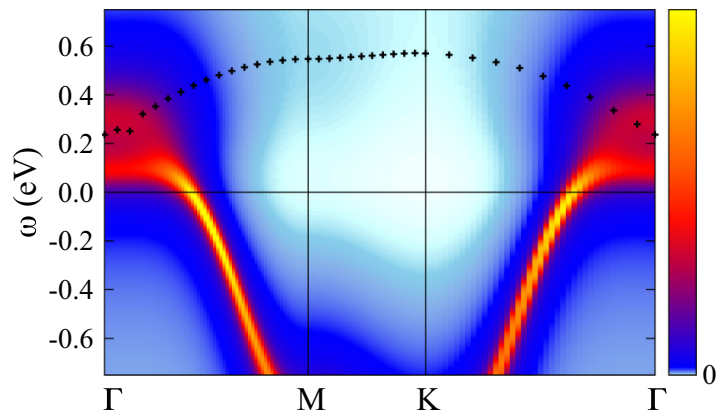

FIG. 3. (Color online) Intensity plot of the interacting $k$-resolved $\mathrm{Na}_{x} \mathrm{CoO}_{2}$ spectral function for $A_{\omega}(\mathbf{k})$ (in arbitrary units) for doping $x=0.67$ along the high-symmetry lines through $\Gamma=(0,0), \mathrm{M}=$ $(1 / 2,-1 / 2 \sqrt{3})$, and $K=(2 / 3,0)$ (in units of $2 \pi / a)$. The spinpolaron states close to $\Gamma$ are clearly visible. Dark crosses show the solution for the spin-polaron band in a $t$ - $J$ model.

marginal and depends on the existence of the Van Hove singularity in the band structure. In order to obtain a qualitative description of spin polarons on the present triangular lattice, we consider a numerical solution of the $t-J$ model. The problem becomes tractable by restricting it to a state of a single magnon and an excess charge carrier in the ferromagnetic state denoted $|\mathrm{FM}\rangle: c_{i \sigma}^{\dagger} S_{j}^{-}|\mathrm{FM}\rangle\left(S_{j}^{-}\right.$lowers the spin at site $j$ by 1). This allows us to compute the dispersion $E(\mathbf{k})$ of the resulting bound state numerically (see the Appendix for details).

In Fig. 3 we display the momentum-resolved DF spectral function for $x=0.67$ together with the model spin-polaron dispersion $E(\mathbf{k})$. We see an overall moderate bandwidth renormalization in DF due to the high doping level. The band structure is very similar to the one obtained within a three-band DMFT description [24] within $0.1 \mathrm{eV}$ of the Fermi level. Above these scattering states near $\Gamma$, we recognize the sideband of antibound states, which we interpret as spinpolaron excitations. This band is not present in the three-band DMFT study of Ref. [24]. As one moves away from $\Gamma$, the spectral weight of the realistic calculation diminishes, but clearly extends in the same direction as the model dispersion. Despite the simplicity of the model, the qualitative description of the spin-polaron excitations through the $t-J$ model calculation is quite remarkable. Given its rudimentary nature, we did not attempt to fit the value of the effective exchange $J$. Nevertheless, the value of $J=0.1|t|=17.8 \mathrm{meV}$ we used here has the same order of magnitude and is quite close to experimental results. Spin-model analyses of inelastic neutron data for $\mathrm{Na}_{x} \mathrm{CoO}_{2}$ at high $x$ yield $J \sim 5-6 \mathrm{meV}$ for the intralayer ferromagnetic spin coupling $[39,40]$. Note that the effective $J$ is not given by the (AFM) superexchange $-4 t^{2} / U$ valid in the spin- $\frac{1}{2}$ Heisenberg limit of the Hubbard model close to half filling.

In order to better understand these results, it is instructive to scrutinize the mechanism by which the spin polarons emerge in the DF calculation. We recall that the self-energy, Eq. (3), has the form of a convolution: $\tilde{\Sigma}_{\omega}(\mathbf{k})=\sum_{\nu \mathbf{q}} \tilde{G}_{\omega+\nu}(\mathbf{k}+\mathbf{q}) S_{\nu}(\mathbf{q})$, where $S_{v}(\mathbf{q})$ encodes information on the magnetic excitations at momentum q. The dominant contribution to $\Sigma$ near $\Gamma$ (i.e., $\mathbf{k} \approx \mathbf{0}$ ), which is responsible for the antibound states, stems from those terms in the sum over $\mathbf{q}$ for which the product of $\tilde{G}(\mathbf{k}+\mathbf{q})$ and $S(\mathbf{q})$ dominates. Due to the presence of the Van Hove singularity, the density of states is large for $\mathbf{k}+\mathbf{q} \approx \mathbf{0}$, or $|\mathbf{k}| \approx|\mathbf{q}| \approx \mathbf{0}$. The self-energy is thus dominated by spin excitations at $\mathbf{q} \approx \mathbf{0}$ and hence by the previously mentioned collective spin excitations at this wave vector found in the study of Ref. [22].

The antibound states in the many-body calculation hence emerge from ferromagnetic paramagnons. This explains the two most striking aspects of our results: In agreement with the experiment, the sideband is rather broad [33]. The quick decay of these excitations is a result of the fact that the lifetime of both the magnon excitations and the QPs is finite. In the simple model, the excitations have infinite lifetime due to the absence of decay channels. Second, their intensity vanishes quickly when moving away from $\Gamma$. According to the foregoing, this is a direct consequence of the fact that at $x=0.67$ and elevated temperature, well-defined ferromagnetic paramagnons only exist in close vicinity of the $\Gamma$ point [22].

We hence identify the sideband excitation as a spinpolaron excitation originating from scattering between QPs and ferromagnetic magnons. The presence of the Van Hove singularity at the top of the holelike band is crucial, because it effectively reduces the dimensionality of the problem. It provides a large density of charge carriers and brings the system close to a Stoner instability, which therefore exhibits a tendency towards ferromagnetism. Note that for cobaltate, we have $t<0$. For the opposite sign, the Van Hove singularity occurs at the bottom of the band. Thus, the formation of a spin-polaron state of the type described in Ref. [37] is once more a consequence of the particular electronic structure of sodium cobaltate $[15,22,32]$.

\section{CONCLUSIONS}

To summarize, we have investigated the spectral properties of the doped Mott material $\mathrm{Na}_{x} \mathrm{CoO}_{2}$ within a combined firstprinciples many-body description. The low-doping regime is rather well described within DMFT, although dynamical AFM correlations lead to an interplay of Slater and Hubbard physics and increased coherence of the high-energy excitations. For higher doping we found sideband excitations at $\omega \sim 0.35 \mathrm{eV}$ in close agreement with optics experiments. By separating spin and charge contributions and taking into account the structure of the self-energy correction to DMFT, we were able to identify these as spin-polaron excitations. This interpretation is supported by a much simpler calculation of the spin-polaron dispersion based on the $t$ - $J$ model. In the material, the excitations result from bound states of QPs with ferromagnetic paramagnons. To our knowledge, this is the first theoretical description of spin-polaron states emerging from dynamical ferromagnetism. The physics described here goes well beyond a simple RPA (random-phase approximation)-like approach, which would merely give rise to an instability towards static Stoner splitting of the bands. The presence of the extended Van Hove singularity in the realistic tight-binding spectrum is crucial for this effect. These excitations may be responsible for non-Fermi liquid behavior observed in this compound at large doping $x \sim 0.71$ [41]. We note that the impact of magnetic fluctuations onto charge transport in sodium cobaltate has 
already been described in a simplified model picture in Ref. [42]. The spin-polaron physics revealed by us is qualitatively different from spin(-orbital) polarons as used by Khaliullin et al. [43] to describe strongly renormalized bands in the occupied part of the spectrum of strongly doped sodium cobaltate. There, orbital-dependent scattering involving the onsite Hund's $J_{H}$ is a vital ingredient. Here the much smaller intersite $J$ is the driving force for the formation of the spin polarons. It is worthwhile to further study these many-body quasibound states theoretically and experimentally. The latter may be accomplished by high-resolution inverse photoemission experiments or QP-scattering analysis within scanning-tunneling microscopy.

\section{ACKNOWLEDGMENTS}

We would like to thank A. N. Rubtsov, S. Brener, and L. Boehnke for helpful discussions. This work has been supported by the DFG-FOR1346 program. M.I.K. acknowledges financial support from ERC (Project No. 338957 FEMTO/NANO) and from NWO via the Spinoza Prize, and H.H. acknowledges financial support from the FP7/ERC, under Grant Agreement No. 278472-MottMetals. Computations have been performed using high-performance computing resources at the NIC, Forschungszentrum Jülich, under Project No. HHH14. The impurity solver [28] and the DF implementation are based on the ALPS libraries [44].

\section{APPENDIX: (ANTI-)BOUND STATES OF ELECTRON AND MAGNON ON ARBITRARY LATTICES}

We consider a ferromagnetic $t-J$ model given by the Hamiltonian

$$
H=\sum_{i j \sigma} t_{i j} c_{i \sigma}^{\dagger} c_{j \sigma}-\frac{1}{2} \sum_{i j} J_{i j} \mathbf{S}_{i} \mathbf{S}_{j},
$$

where $c_{i \sigma}^{(\dagger)}$ destroys (creates) a particle with spin-projection $\sigma$ on the site $i$ only if there is already a particle with opposite spin. $J_{i j}>0$ corresponds to ferromagnetic coupling. We call the state with every site occupied by one electron with spin-up $|\mathrm{FM}\rangle$. In the following, we restrict the investigation to the specific sector $c_{i \sigma}^{\dagger} S_{j}^{-}|\mathrm{FM}\rangle \equiv|i j\rangle$.

With the ansatz

$$
|\Psi\rangle=\sum_{i j, i \neq j} \psi_{i j}|i j\rangle
$$

and the eigenvalue equation $H|\Psi\rangle=E|\Psi\rangle$ it follows for the coefficients $\psi_{i j}$ [37]

$$
E \psi_{i j}=\sum_{k \neq j} t_{i k} \psi_{k j}+t_{i j} \psi_{j i}+\sum_{k \neq i} J_{j k}\left(\psi_{i j}-\psi_{i k}\right) .
$$

From the definition of $|i j\rangle$ it is clear that $|i i\rangle=0$ holds. We therefore define $\psi_{i i}=0$. With the Fourier expansion of the form

$$
\begin{array}{r}
\psi_{i j}=\sum_{\mathbf{q}} \psi_{\mathbf{q}} \exp \left\{i\left[\mathbf{q} \mathbf{R}_{i}+(\mathbf{Q}-\mathbf{q}) \mathbf{R}_{j}\right]\right\}, \\
\psi_{\mathbf{q}}=\sum_{i j} \psi_{i j} \exp \left\{-i\left[\mathbf{q} \mathbf{R}_{i}+(\mathbf{Q}-\mathbf{q}) \mathbf{R}_{j}\right]\right\},
\end{array}
$$

we restrict the solutions to the subspace of electron-magnon pairs propagating with the wave vector $\mathbf{Q}$. Transforming (A3) via (A5) leads to [37]

$$
\begin{aligned}
& \left(E-J_{0}-t_{\mathbf{q}}+J_{\mathbf{Q}-\mathbf{q}}\right) \psi_{\mathbf{q}} \\
& \quad=\sum_{\mathbf{p}}\left(t_{\mathbf{Q}-\mathbf{q}-\mathbf{p}}-t_{\mathbf{p}}+J_{\mathbf{Q}-\mathbf{p}}-J_{\mathbf{q}-\mathbf{p}}\right) \psi_{\mathbf{p}},
\end{aligned}
$$

with $J_{0}=J_{\mathbf{q}=\mathbf{0}}$. This is a Fredholm integral equation of the second kind with separable integral kernel (if we take into account the hopping and exchange to a finite number of neighbors in real space). To solve that integral equation, we separate the kernel

$$
K_{\mathbf{q p}}=t_{\mathbf{Q}-\mathbf{q}-\mathbf{p}}-t_{\mathbf{p}}+J_{\mathbf{Q}-\mathbf{p}}-J_{\mathbf{q}-\mathbf{p}}
$$

by rewriting it as

$$
\begin{aligned}
K_{\mathbf{q p}}=\sum_{\mathbf{R}}\left[t_{\mathbf{R}}\left(e^{i(\mathbf{Q}-\mathbf{q}-\mathbf{p}) \mathbf{R}}-e^{i \mathbf{p R}}\right)\right. \\
\left.+J_{\mathbf{R}}\left(e^{i(\mathbf{Q}-\mathbf{p}) \mathbf{R}}-e^{i(\mathbf{q}-\mathbf{p}) \mathbf{R}}\right)\right] \\
=\sum_{\mathbf{R}}\left[t_{\mathbf{R}}\left(e^{i(-\mathbf{Q}+\mathbf{q}) \mathbf{R}}-1\right)\right. \\
\left.+J_{\mathbf{R}}\left(e^{-i \mathbf{Q R}}-e^{-i \mathbf{q} \mathbf{R}}\right)\right] e^{i \mathbf{p R}} \\
=\sum_{\mathbf{R}} N_{\mathbf{R}}(\mathbf{p}) M_{\mathbf{R}}(\mathbf{q}),
\end{aligned}
$$

where

$$
N_{\mathbf{R}}(\mathbf{p})=e^{i \mathbf{p R}},
$$

$$
M_{\mathbf{R}}(\mathbf{q})=t_{\mathbf{R}}\left(e^{i(-\mathbf{Q}+\mathbf{q}) \mathbf{R}}-1\right)+J_{\mathbf{R}}\left(e^{-i \mathbf{Q R}}-e^{-i \mathbf{q} \mathbf{R}}\right) .
$$

After dividing (A6) by the factor on the left-hand side, i.e.,

$$
\psi_{\mathbf{q}}=\sum_{\mathbf{R}} \frac{M_{\mathbf{R}}(\mathbf{q})}{E-J_{0}-t_{\mathbf{q}}+J_{\mathbf{Q}-\mathbf{q}}} \sum_{\mathbf{p}} N_{\mathbf{R}}(\mathbf{p}) \psi_{\mathbf{p}},
$$

we multiply both sides with $N_{\mathbf{R}^{\prime}}(\mathbf{q})$ and sum over $\mathbf{q}$ :

$$
\begin{aligned}
\sum_{\mathbf{q}} N_{\mathbf{R}^{\prime}}(\mathbf{q}) \psi_{\mathbf{q}}= & \sum_{\mathbf{R}} \sum_{\mathbf{q}} \frac{N_{\mathbf{R}^{\prime}}(\mathbf{q}) M_{\mathbf{R}}(\mathbf{q})}{E-J_{0}-t_{\mathbf{q}}+J_{\mathbf{Q}-\mathbf{q}}} \\
& \times \sum_{\mathbf{p}} N_{\mathbf{R}}(\mathbf{p}) \psi_{\mathbf{p}} .
\end{aligned}
$$

This can be written in the form

$$
c_{\mathbf{R}^{\prime}}=\sum_{\mathbf{R}} a_{\mathbf{R}^{\prime} \mathbf{R}} c_{\mathbf{R}},
$$

with

$$
\begin{gathered}
c_{\mathbf{R}}=\sum_{\mathbf{q}} N_{\mathbf{R}}(\mathbf{q}) \psi_{\mathbf{q}}, \\
a_{\mathbf{R}^{\prime} \mathbf{R}}=\sum_{\mathbf{q}} \frac{N_{\mathbf{R}^{\prime}}(\mathbf{q}) M_{\mathbf{R}}(\mathbf{q})}{E-J_{0}-t_{\mathbf{q}}+J_{\mathbf{Q}-\mathbf{q}}},
\end{gathered}
$$

or, in matrix form,

$$
[\mathbb{1}-A(E)] \mathbf{C}=0,
$$


where $A_{i j}=a_{\mathbf{R}_{i} \mathbf{R}_{j}}$ and $C_{i}=c_{\mathbf{R}_{i}}$. The dispersion $E_{\mathbf{Q}}$ of the spin-polaron band for given $\mathbf{Q}$ is determined by solving $\operatorname{det}[\mathbb{1}-A(E)]=0$ numerically for the energy $E_{\mathbf{Q}}$ and with the constraint $\left|E_{\mathbf{Q}}-J_{0}\right|>-t_{\mathbf{q}}+J_{\mathbf{Q}-\mathbf{q}}$.

In $1 \mathrm{D}$, an exact solution exists [37]:

$$
E^{(1 \mathrm{D})}(k)=-\frac{2 t[t-J \cos (k)]}{|t|+J} .
$$

For the 2D case, we have solved Eq. (A17) on the triangular lattice. We replace $t_{i j}$ with the values $t, t^{\prime}$, and $t^{\prime \prime}$ given in the main text as appropriate. We further use an effective exchange constant $J_{i j}=J>0$ for $i$ and $j$ NNs and $J_{i j}=0$ otherwise. The resulting matrix has size $18 \times 18$. The number 18 is due to the fact that the sum in (A14) is over the three shells of nearest-, next-nearest, and third-nearest neighbors, which on the triangular lattice contain six atoms each.
[1] K. Takada, H. Sakurai, E. Takayama-Muromachi, F. Izumi, R. A. Dilanian, and T. Sasaki, Nature (London) 422, 53 (2003).

[2] I. R. Mukhamedshin, H. Alloul, G. Collin, and N. Blanchard, Phys. Rev. Lett. 94, 247602 (2005).

[3] G. Lang, J. Bobroff, H. Alloul, G. Collin, and N. Blanchard, Phys. Rev. B 78, 155116 (2008).

[4] M. L. Foo, Y. Wang, S. Watauchi, H. W. Zandbergen, T. He, R. J. Cava, and N. P. Ong, Phys. Rev. Lett. 92, 247001 (2004).

[5] J. Sugiyama, H. Itahara, J. H. Brewer, E. J. Ansaldo, T. Motohashi, M. Karppinen, and H. Yamauchi, Phys. Rev. B 67, 214420 (2003).

[6] T. Motohashi, R. Ueda, E. Naujalis, T. Tojo, I. Terasaki, T. Atake, M. Karppinen, and H. Yamauchi, Phys. Rev. B 67, 064406 (2003).

[7] A. T. Boothroyd, R. Coldea, D. A. Tennant, D. Prabhakaran, L. M. Helme, and C. D. Frost, Phys. Rev. Lett. 92, 197201 (2004).

[8] I. Terasaki, Y. Sasago, and K. Uchinokura, Phys. Rev. B 56, R12685 (1997).

[9] D. Singh, Phys. Rev. B 61, 13397 (2000).

[10] M. Z. Hasan, Y.-D. Chuang, D. Qian, Y. W. Li, Y. Kong, A. Kuprin, A. V. Fedorov, R. Kimmerling, E. Rotenberg, K. Rossnagel, Z. Hussain, H. Koh, N. S. Rogado, M. L. Foo, and R. J. Cava, Phys. Rev. Lett. 92, 246402 (2004).

[11] T. Kroll, A. A. Aligia, and G. A. Sawatzky, Phys. Rev. B 74, 115124 (2006).

[12] A. Georges, G. Kotliar, W. Krauth, and M. J. Rozenberg, Rev. Mod. Phys. 68, 13 (1996).

[13] S. Y. Li, L. Taillefer, D. G. Hawthorn, M. A. Tanatar, J. Paglione, M. Sutherland, R. W. Hill, C. H. Wang, and X. H. Chen, Phys. Rev. Lett. 93, 056401 (2004).

[14] H. Ohta, K. Yoshimura, Z. Hu, Y. Y. Chin, H.-J. Lin, H. H. Hsieh, C. T. Chen, and L. H. Tjeng, Phys. Rev. Lett. 107, 066404 (2011).

[15] F. Lechermann, Phys. Rev. Lett. 102, 046403 (2009).

[16] Lattice quantum Monte Carlo simulations are not applicable here due to the sign problem.

[17] A. N. Rubtsov, M. I. Katsnelson, and A. I. Lichtenstein, Phys. Rev. B 77, 033101 (2008).

[18] A. N. Rubtsov, M. I. Katsnelson, and A. I. Lichtenstein, Ann. Phys. 327, 1320 (2012).

[19] A. Toschi, A. A. Katanin, and K. Held, Phys. Rev. B 75, 045118 (2007).

[20] O. I. Motrunich and P. A. Lee, Phys. Rev. B 69, 214516 (2004).

[21] J. O. Haerter, M. R. Peterson, and B. S. Shastry, Phys. Rev. Lett. 97, 226402 (2006).

[22] L. Boehnke and F. Lechermann, Phys. Rev. B 85, 115128 (2012).

[23] M. M. Korshunov, I. Eremin, A. Shorikov, V. I. Anisimov, M. Renner, and W. Brenig, Phys. Rev. B 75, 094511 (2007).
[24] L. Boehnke and F. Lechermann, Phys. Status Solidi A 211, 1267 (2014).

[25] We choose the experimentally determined value [10], although the interaction would have to be renormalized because of the downfolding to a single band. Because the material is in the large $U / W$ regime, we do not expect our result to sensitively depend on the value of $U$.

[26] H. Hafermann, G. Li, A. N. Rubtsov, M. I. Katsnelson, A. I. Lichtenstein, and H. Monien, Phys. Rev. Lett. 102, 206401 (2009).

[27] H. Hafermann, E. G. C. P. van Loon, M. I. Katsnelson, A. I. Lichtenstein, and O. Parcollet, Phys. Rev. B 90, 235105 (2014).

[28] H. Hafermann, P. Werner, and E. Gull, Comput. Phys. Commun. 184, 1280 (2013).

[29] E. Gull, A. J. Millis, A. I. Lichtenstein, A. N. Rubtsov, M Troyer, and P. Werner, Rev. Mod. Phys. 83, 349 (2011).

[30] H. Hafermann, K. R. Patton, and P. Werner, Phys. Rev. B 85 , 205106 (2012).

[31] H. Hafermann, F. Lechermann, A. N. Rubtsov, M. I. Katsnelson, A. Georges, and A. I. Lichtenstein, in Modern Theories of ManyParticle Systems in Condensed Matter Physics, edited by D. C. Cabra, A. Honecker, and P. Pujol, Lecture Notes in Physics Vol. 843 (Springer Berlin Heidelberg, 2012), pp. 145-214.

[32] O. E. Peil, A. Georges, and F. Lechermann, Phys. Rev. Lett. 107, 236404 (2011).

[33] N. L. Wang, P. Zheng, D. Wu, Y. C. Ma, T. Xiang, R. Y. Jin, and D. Mandrus, Phys. Rev. Lett. 93, 237007 (2004).

[34] S. Methfessel and D. C. Mattis, Handbuch der Physik (Springer, Berlin, 1968), Vol. 18.

[35] B. S. Shastry and D. C. Mattis, Phys. Rev. B 24, 5340 (1981).

[36] M. I. Auslender, V. Y. Irkhin, and M. I. Katsnelson, J. Phys. C: Solid State Phys. 17, 669 (1984).

[37] M. I. Katsnelson, Fiz. Met. Metalloved. 54, 396 (1982).

[38] Y. Nagaoka, Phys. Rev. 147, 392 (1966). V. Y. Irkhin and M. I. Katsnelson, J. Phys. C 18, 4173 (1985).

[39] S. P. Bayrakci, I. Mirebeau, P. Bourges, Y. Sidis, M. Enderle, J. Mesot, D. P. Chen, C. T. Lin, and B. Keimer, Phys. Rev. Lett. 94, 157205 (2005).

[40] L. M. Helme, A. T. Boothroyd, R. Coldea, D. Prabhakaran, A Stunault, G. J. McIntyre, and N. Kernavanois, Phys. Rev. B 73, 054405 (2006).

[41] L. Balicas, Y. J. Jo, G. J. Shu, F. C. Chou, and P. A. Lee, Phys. Rev. Lett. 100, 126405 (2008).

[42] B. Liu, Y. Liang, S. Feng, and W. Y. Chen, Phys. Rev. B 69 , 224506 (2004).

[43] G. Khaliullin and J. Chaloupka, Phys. Rev. B 77, 104532 (2008).

[44] B. Bauer et al., J. Stat. Mech.: Theory Exp. (2011) P05001. 